\title{
Effect of ID ACE gene polymorphism on dietary composition and obesity-related anthropometric parameters in the Czech adult population
}

\author{
Julie Bienertova-Vasku • Petr Bienert $\cdot$ Lenka Sablikova $\cdot$ Lenka Slovackova \\ Martin Forejt · Zlata Piskackova · Lenka Kucerova · Katerina Heczkova • \\ Zuzana Brazdova $\cdot$ Anna Vasku
}

Received: 18 April 2009/ Accepted: 22 June 2009/Published online: 16 July 2009

(C) Springer-Verlag 2009

\begin{abstract}
The aim of this study was to investigate the possible associations between insertion/deletion (ID) polymorphism in angiotensin-converting enzyme (ACE) (dbSNP rs 4646994) with the food intake and body composition in the Czech non-obese, obese and extremely obese populations. A total of 453 various-weighted individuals were enrolled in the study and were according to their BMI assigned into following subgroups, such as obese $(30 \leq$ BMI $<40)$, morbidly obese (BMI $\geq 40)$ and nonobese $(20<\mathrm{BMI}<30)$ subjects. Both the obese cases and the non-obese controls underwent the identical subset of standardized examinations (BMI, \% body fat, waist-to-hip ratio, skin fold thickness, native dietary composition examined by 7-day food records, etc.). No significant casecontrol differences in genotype distributions or allelic frequencies were observed. There were no differences in genotype frequencies between males and females either. The prevalence of obesity was significantly higher among subjects with the II genotype (42\%) when compared with those with DD (36\%) and those with ID (37\%) genotypes $(P=0.04)$. When compared with carbohydrate intake in the whole studied cohort, the odds ratios of carrying the DD allele in the morbidly obese cohort were 0.84 (95\% CI 0.34 , $2.10, P=0.17), 0.27(0.07,0.98, P=0.02)$, and $4.25(1.44$,
\end{abstract}

J. Bienertova-Vasku $(\bowtie) \cdot$ P. Bienert · L. Sablikova ·

L. Slovackova $\cdot$ A. Vasku

Department of Pathological Physiology, Medical Faculty,

Masaryk University, Kamenice 5, 62500 Brno, Czech Republic

e-mail: jbienert@med.muni.cz; Vasku.Julie@seznam.cz

M. Forejt · Z. Piskackova $\cdot$ L. Kucerova $\cdot$ K. Heczkova ·

Z. Brazdova

Department of Preventive Medicine, Faculty of Medicine,

Masaryk University, Tomesova 12, 60200 Brno,

Czech Republic
$12.51, P=0.005$ ) in those individuals consuming $<210$, 210-260, and $>260 \mathrm{~g}$ of carbohydrates/day, respectively. Based on our findings, the ID ACE polymorphism could represent a gene modulator of carbohydrate intake in morbidly obese Czech population; the strong significant effect of DD genotype was observed in the phenotypes of extreme obesity with the highest carbohydrate intake.

Keywords ID ACE · Polymorphism · Obesity · Food intake

\section{Introduction}

In Western countries, overweight and obesity (OMIM 601665) prevalence has been steadily increasing for decades. It is generally suggested that this is mainly due to typical environmental factors, such as high-fat diet or lack of physical activity [31]. Without question, the common obesity phenotypes are indisputably associated with typical lifestyle and dietary characteristics that are superposed on the genetic background of an individual [10]. However, the individual response to environmental stimuli varies substantially between individuals across the population, and to the present date, little is known about the variations of the genetic factors underlying the susceptibility to specific eating behavior.

The control of eating behavior in mammals comprises the complex network of hormones and brain circuits that regulate food intake and energy expenditure. However, although numerous types of knockout and transgenic animals have provided insight into the role of specific hormones and neurotransmitters on the regulation of body weight, the roles of genetic predisposition in controlling the eating behavior in humans are elusive. 
Several studies have reported a heritable component for eating behavior [8, 9, 17, 29]. Both the meal size and meal frequency are the two traits expressing heritability [10, 31]. Only a few studies have been conducted to find genes underlying this heritability. Two studies have reported on genome-wide linkage screens: Steinle et al. [29] showed specific logarithm of odds scores for specific eating habits and Bouchard et al. [3] reported evidence of a specific candidate gene for eating behavior patterns and predisposition to obesity. Although a vast number of candidate genes were reported to be associated with obesity, their interactions and possible different magnitude of their effects complicate substantially the interpretation of the results.

Several lines of evidence have previously suggested that angiotensin II, the direct consequence of ACE activity, impairs insulin sensitivity [22, 24], and insulin resistance promotes the development of various co-morbidities by upregulating the number and activity of angiotensin II receptors [20]. In several other studies, insulin resistance has been improved in response to treatment with angiotensin I-converting enzyme inhibitors (ACEIs) [12, 13]. Angiotensin II receptor blockers (ARB) and ACEIs have both been recommended as first lines of antihypertensive treatment in patients with diabetes, but it remains rather elusive whether the insulin action responds as favorably to the former as it does to the latter. The association between excess adiposity and insulin resistance is well established in both children and adults, and excess adiposity is clearly an important antecedent factor in the development of insulin resistance [11]. However, it has to be also mentioned that impaired glucose homeostasis may itself be a factor contributing to subsequent weight gain thus maintaining the vicious cycle of excessive weight gain. The hypothesis that circulating insulin provides a physiologic signal to brain areas involved in energy homeostasis is implying a mechanism whereby this peptide traverses the blood-brain barrier (BBB) to enter cerebrospinal fluid [21]. Earlier studies have demonstrated that, during intravenous infusion, increases in plasma insulin are paralleled by proportionate (albeit much smaller in absolute terms) increases in cerebrospinal fluid insulin levels in humans [32]. Subsequent studies showed that this insulin uptake process involves a saturable transport mechanism [1], and, this is combined with the findings that insulin receptors are expressed by endothelial cells in brain and that these receptors are capable of transendothelial insulin transport, which led to the conclusion that insulin delivery across the BBB involves an active, receptor-mediated mechanism [11]. Thus, we hypothesized that impaired insulin sensitivity due to disturbances in RAS could consecutively lead to various effects at the level of central nervous system too.

The common insertion/deletion polymorphism (ID ACE; rs 4646994) in the gene for angiotensin-converting enzyme (ACE) was previously associated with obesity [3, 15, 19, 30], both in preschoolers, adolescents and adults. Previously, it has been also reported that approximately $47 \%$ of the variance in plasma ACE activity can be explained by this polymorphism located in intron 16 of the ACE gene, the D allele being associated with increased ACE activity [25]. Given its intronic location, it is highly unlikely that the ID polymorphism would represent a functional variant and it is much more likely that these associations are mediated through linkage disequilibrium with a neighboring functional variant. However, the ID polymorphism is associated with ACE activity and represents a simply testable marker for any functional polymorphism in its close vicinity [14]. Recently, significant interactions were reported to be found between the ACE I/D polymorphism and total energy intake on waist circumference ( $P=0.004$ for interaction) and the ACE I/D polymorphism and protein intake on BMI and being overweight $(P<0.05$ for interaction) in Greek toddlers and preschoolers [14]. Furthermore, it was reported in this study that the ACE I/D polymorphism may in children modify the effect of fat intake on waist circumference and BMI, but this interaction disappeared after the adjustment for additional potential confounders. However, no such study focusing on anthropometric and dietary characteristics has been so far conducted on a large population sample of adults.

Therefore, the aim of the present study was to investigate possible associations of ID ACE polymorphism with the dietary composition (i.e. total energy, fat, protein and carbohydrate intake, fiber intake, cholesterol intake, etc.) and obesity-related phenotypes among Czech (middleEuropean) Caucasian adult population.

\section{Materials and methods}

\section{Subjects}

A total of 453 unrelated Czech Caucasian individuals were recruited for this study in a masmedial campaign addressing the population of south Moravia region of the Czech Republic [2] and were divided into three groups. The inclusion and exclusion criteria were derived from $\mathrm{Ma}$ et al. [18]. To sum up, the participation was limited to individuals who were (1) not taking cholesterol-loweringor weight-controlling medications, (2) not currently on lipid-lowering- or weight-control diets, (3) free from possible causes of secondary hypercholesterolemia (e.g. pregnancy), and (4) free from severe chronic illness (e.g. cancer, renal failure, and heart failure). The study was approved by the Committee for Ethics of Medical Experiments on Human Subjects, Faculty of Medicine, Masaryk University, Brno and was performed in adherence to the 
Declaration of Helsinki Guidelines. Participants gave their written informed consent before they entered the study and this consent was archived.

The first group consisted of 207 obese individuals $\left(40>\mathrm{BMI} \geq 30\right.$; mean BMI $34.4 \pm 2.8 \mathrm{~kg} / \mathrm{m}^{2}$, mean age $49.1 \pm 11.6$ years). Another set of 74 morbidly obese patients were enrolled to the study (BMI $\geq 40$; mean BMI $45.8 \pm 5.6 \mathrm{~kg} / \mathrm{m}^{2}$, mean age $51.3 \pm 11.0$ years). The control group consisted of 172 healthy non-obese control subjects with no history of childhood obesity or eating disorder $\left(20<\mathrm{BMI}<30\right.$; mean BMI $25.2 \pm 3.2 \mathrm{~kg} / \mathrm{m}^{2}$, mean age $38.7 \pm 13.4$ years).

Data on personal or family history of obesity, birth weight, age at onset of obesity, eating disorders, age of menarche and menopause in women, family history of sterility, infertility or stillbirth were obtained by a professional using a semi-structured interview. A positive family history for obesity was estimated as one obese relative with $\mathrm{BMI} \geq 30 \mathrm{~kg} / \mathrm{m}^{2}$ in the close family (siblings, parents and their siblings, and grandparents). Both the obese cases and the non-obese controls underwent the same subset of examination focused on their anthropometric characteristics, dietary intake and genetic background of the individual and the family.

\section{Anthropometric characteristics}

All phenotypic measurements were performed by a specialist and included weight, height, BMI, lean body mass, total body fat, waist, and hip circumferences, waist-to-hip ratio (WHR), skin fold thickness. Body composition was assessed by bioelectrical impedance analysis, using the single frequency bio-impedance analyzer BodyStat (Bodystat Ltd, Douglas, Isle of Man, UK) with the subject lying in a supine position.

\section{Dietary intake}

The dietary habits of the subjects were assessed with a modified diet history method as described by Larsson et al. [16] which consider the entire diet, including cooking methods. The employed method combined quantitative and semiquantitative measurements of dietary intake, using a combination of a food frequency questionnaire, which surveys the regularly consumed foods during last 7 days. For each food item, usual intake frequency and portion size were given. Portion sizes were estimated using a booklet with pictures of the different food items with varying portion sizes. All cooked meals and beverages during 7 days were recorded in the menu book, including all ingredients of each meal. At the start of the study, the subjects were instructed individually by a dietician how to fill out the questionnaires. Two to 3 weeks later, the subjects returned to the dietician individually and their eating habits were consulted.

The dieticians recorded the usual amount consumed by the subject of each food item in the food frequency questionnaire and the 7-day food record. Food intake date obtained from the study subjects were analyzed and energy and nutrient intake were calculated using the Nutrimaster Diet Analysis software (Abbott Laboratories, Abbott Park, IL, USA) adjusted on the Czech population. Special attention was paid to extreme snacking behavior, dieting, extreme portion sizes, and irregularity in eating.

Selected parameters were followed, including: total energy intake per day; percent of energy intake in fat, carbohydrate, and protein; percent of total energy intake per day and servings per day for each food group; cholesterol, saturated fat, and fiber intake; and grams of carbohydrates per day and percent of energy derived from carbohydrates.

\section{Genotyping}

DNA for analyses was extracted from $5 \mathrm{~mL}$ of the patients' saliva collected after $3 \mathrm{~h}$ of fasting. Genotyping of the ID ACE polymorphism was performed as described previously [26], with a subsequent verification of the DD genotype by a method described previously [28]. Using gel electrophoresis, the polymerase chain reaction (PCR) products were distinguished as a $190 \mathrm{bp}$ fragment in the absence and a $490 \mathrm{bp}$ fragment in the presence of the insertion (genotypes described as II-490 bps, ID-490 + 190 bps, DD-190 bps).

\section{Statistics}

The observed number of each genotype was compared with that expected for a population in Hardy-Weinberg equilibrium using $\chi^{2}$ test. Fisher's exact test with TukeyKramer's method of adjustment for multiple comparisons was employed for comparison of categorical variables. Differences between continuous variables were evaluated using analysis of variance (ANOVA), an analysis of covariance (ANCOVA) with corresponding post hoc test for more than two groups. Univariate logistic regression analysis was performed to evaluate the influence of the genotype and other variables obesity development or its associated features. In the logistic regression analysis, the genotypes were coded as quantitative variables ( $\mathrm{II}=0$, $\mathrm{ID}=1, \mathrm{DD}=2$ )

Using sample tertiles, the nutrient variables were categorized in three groups of equal size (the upper third, the middle third and the lower third) as described by Santos et al. [27]. Each nutrient variable was then included in logistic regressions as binary indicators leaving one category as the reference. 
Multiple regression analysis was applied in all cases of associations between the genotypes and clinical parameters, significant in the preceding univariate analysis. The data analysis was performed using Statistica v. 8.0 (Statsoft Inc., Tulsa, OK, USA) program package at the significance level defined as $P<0.05 \%$.

\section{Results}

The baseline demographic, anthropometric, dietary and clinical characteristics of the study subjects in relation to gender are listed in Table 1. No significant differences both in genotype distributions and allele frequencies were observed when comparing the morbidly obese cohort (BMI $\geq 40)$ and the obese cohort $(30 \leq \mathrm{BMI}<40)$ with the controls $(20<\mathrm{BMI}<30)$. The entire investigated population as well as the subgroups were found to be in HardyWeinberg equilibrium at the ID ACE locus and the overall allele frequencies in the entire cohort were calculated as $f(\mathrm{I})=48.3 \%$ and $f(\mathrm{D})=51.7 \%$. The genotype frequencies in the total study cohort were 30,44 , and $26 \%$ for DD, DI, and II, respectively. There were no differences in genotype frequencies between males and females. The prevalence of obesity was significantly higher among subjects with the II genotype (42\%) when compared with those with DD $(36 \%)$ and those with ID $(37 \%)$ genotypes $(P=0.04)$.

Effect of ID ACE on anthropometric and clinical characteristics (BMI, WHR, total body fat, skin fold thickness, blood pressure)

In the next step, we tested whether these SNPs had any effect on anthropometric characteristics related to obesity in the study subjects. A significant decrease in the percentage of body water was observed among DD homozygotes $(45.2 \%)$ against the ID $(46.2 \%)$ and II $(48.3 \%)$ carriers $(P=0.02)$. Moreover, the DD homozygotes presented with the highest thickness of the supraspinal skin fold (average thickness $25 \mathrm{~mm}$ ) when compared with the ID $(23 \mathrm{~mm})$ and II $(20 \mathrm{~mm})$ carriers $(P=0.02)$.

In the multivariate regression modeling across all the study subjects, the ID ACE polymorphism did not express (after adjustment for age and sex) a prediction role on any of the investigated parameters, such as BMI, total body fat, total body water, waist circumference, hip circumference, WHR, total body fat.

In the next step, we tested whether I or D allele expresses any effect on systolic/diastolic blood pressure in relation to investigated dietary composition. The associations of total carbohydrate intake with diastolic blood pressure by ID ACE are summarized in Table 2.

Effect of SNPs on dietary characteristics and dietary composition of the study subjects

In the univariate regression modeling, the examined polymorphism did not serve as an independent predictor for percentage of daily energy intake from macronutrients or abnormal eating patterns (extreme snacking behavior, extreme portion sizes, night eating, and irregular food intake). When compared with carbohydrate intake in the whole studied cohort, the odds ratios of carrying the DD allele in the morbidly obese cohort were 0.84 [95\% confidence interval (CI): $0.34,2.10, P=0.17], 0.27(0.07$, $0.98, P=0.02)$, and $4.25(1.44,12.51, P=0.005)$ in those individuals consuming $<210,210-260$, and $>260 \mathrm{~g}$ of carbohydrates/day, respectively (Table 3). However, the

Table 1 Baseline patients' characteristics

\begin{tabular}{|c|c|c|c|c|c|c|}
\hline \multirow{2}{*}{$\begin{array}{l}\text { Variable } \\
\text { Body composition }\end{array}$} & \multicolumn{2}{|l|}{ Obese } & \multicolumn{2}{|l|}{ Morbidly obese } & \multicolumn{2}{|l|}{ Controls } \\
\hline & Females & Males & Females & Males & Females & Males \\
\hline$N$ & 147 & 60 & 59 & 15 & 131 & 41 \\
\hline Age (years) & $49.9 \pm 11.2$ & $47.1 \pm 12.5$ & $51.8 \pm 10.7$ & $49.2 \pm 12.2$ & $39 \pm 13.3$ & $37.8 \pm 13.9$ \\
\hline BMI $\left(\mathrm{kg} / \mathrm{m}^{2}\right)$ & $34.3 \pm 2.8$ & $34.5 \pm 2.9$ & $45.6 \pm 5.6$ & $46.9 \pm 5.5$ & $25 \pm 3.4$ & $26 \pm 2.5$ \\
\hline Body fat (\%) & $43.7 \pm 4.7$ & $29.7 \pm 6.4$ & $52.5 \pm 4.1$ & $41.8 \pm 3.7$ & $31.3 \pm 7.2$ & $19.8 \pm 6.1$ \\
\hline \multicolumn{7}{|l|}{ Dietary intake } \\
\hline Energy (MJ) & $7,921 \pm 2,620$ & $10,548 \pm 2,724$ & $7,687 \pm 2,099$ & $10,283 \pm 3,589$ & $7,828 \pm 1,853$ & $10,659 \pm 2,395$ \\
\hline Protein (\% energy) & $69.7 \pm 21.3$ & $88.2 \pm 21.2$ & $70 \pm 18.9$ & $88.3 \pm 36.9$ & $64.8 \pm 16.9$ & $82.5 \pm 21.1$ \\
\hline Carbohydrates (\%) energy) & $230.2 \pm 74.7$ & $303 \pm 89.3$ & $223.4 \pm 63.6$ & $270.5 \pm 82$ & $234.8 \pm 59.8$ & $312.8 \pm 84.3$ \\
\hline Fat (\% energy) & $74 \pm 30.8$ & $97.6 \pm 29.5$ & $72.8 \pm 24.6$ & $95.9 \pm 38.6$ & $72.3 \pm 21.3$ & $97.7 \pm 26.5$ \\
\hline Adiponectin $(\mu \mathrm{g} / \mathrm{mL})$ & $3.5 \pm 1.5$ & $3.3 \pm 0.5$ & $9.6 \pm 5.2$ & $8.8 \pm 7$ & $9.4 \pm 5.6$ & $5.3 \pm 2.7$ \\
\hline
\end{tabular}

Values are expressed as mean $\pm \mathrm{SD}$, morbidly obese $\left(\mathrm{BMI} \geq 40 \mathrm{~kg} / \mathrm{m}^{2}\right)$, obese $\left(30 \leq \mathrm{BMI}<40 \mathrm{~kg} / \mathrm{m}^{2}\right)$, non-obese controls $\left(20<\mathrm{BMI}<30 \mathrm{~kg} / \mathrm{m}^{2}\right)$ 
Table 2 Standardized $\beta$ coefficients (95\% confidence intervals) for associations of total dietary carbohydrate intake with diastolic blood pressure by ID ACE polymorphism: multivariate regression analysis in the total study cohort

\begin{tabular}{|c|c|c|c|}
\hline \multirow[t]{2}{*}{ Model } & \multicolumn{2}{|c|}{ ID ACE polymorphism } & \multirow[t]{2}{*}{$P$ for interaction ${ }^{\mathrm{a}}$} \\
\hline & II & $\mathrm{ID}+\mathrm{DD}$ & \\
\hline 1 & $0.03(-0.08-0.13)$ & $-0.04(-0.15-0.06)$ & 0.12 \\
\hline 2 & $0.03(-0.09-0.12)$ & $-0.05(-0.15-0.06)$ & 0.07 \\
\hline 3 & $-0.05(-0.05-0.15)$ & $-0.04(-0.14-0.06)$ & 0.12 \\
\hline 4 & $-0.02(-0.15-0.06)$ & $-0.04(-0.15-0.06)$ & 0.11 \\
\hline
\end{tabular}

Model 1: total dietary carbohydrate intake adjusted for age, sex, fat and protein intake

Model 2: Model $1+$ fiber intake

Model 3: Model $1+$ waist-to-hip ratio

Model 4: Model $1+$ BMI

a Interaction between total carbohydrate intake and ID ACE polymorphism

same trend was not observed in the obese or non-obese cohort; the analyses of the protein and fat intake did not reveal significant associations either.

In Table 3, stratified multivariate analyses of total carbohydrate intake across the whole study population of 453 individuals in relation to ACE genotypes are presented. None of the presented models adjusted for age, sex, fat, and protein intake (Model 1), plus fiber intake (Model 2) or

Table 3 Relative risk (OR) of ID ACE polymorphism for carbohydrate intake in the total cohort studied and subsets according to BMI

\begin{tabular}{|c|c|c|c|c|}
\hline \multirow{2}{*}{$\begin{array}{l}\text { Intake of } \\
\text { carbohydrates }\end{array}$} & \multicolumn{2}{|l|}{ ID ACE } & \multirow{2}{*}{$\begin{array}{l}\text { Odds ratio } \\
(95 \% \mathrm{CI})\end{array}$} & \multirow[t]{2}{*}{$P$} \\
\hline & $\begin{array}{l}\mathrm{II}+\mathrm{ID} \\
N(\%)\end{array}$ & $\begin{array}{l}\mathrm{DD} \\
N(\%)\end{array}$ & & \\
\hline All subjects & 319 & 134 & 1.0 & \\
\hline$<210$ & 105 & 46 & $1.04(0.70-1.56)$ & 0.08 \\
\hline $210-260$ & 112 & 38 & $0.81(0.53-1.23)$ & $0.05 *$ \\
\hline$>260$ & 102 & 50 & $1.17(0.79-1.73)$ & 0.06 \\
\hline Obese & 146 & 61 & 1.0 & \\
\hline$<210$ & 50 & 22 & $1.05(0.59-1.79)$ & 0.12 \\
\hline $210-260$ & 43 & 18 & $1.00(0.54-1.88)$ & 0.13 \\
\hline$>260$ & 53 & 21 & $0.95(0.53-1.70)$ & 0.12 \\
\hline Morbidly obese & 49 & 25 & 1.0 & \\
\hline$<210$ & 21 & 9 & $0.84(0.34-2.10)$ & 0.17 \\
\hline $210-260$ & 22 & 3 & $0.27(0.07-0.98)$ & $0.02 *$ \\
\hline$>260$ & 6 & 13 & $4.25(1.44-12.51)$ & $0.005 *$ \\
\hline Non-obese & 124 & 48 & 1.0 & \\
\hline$<210$ & 34 & 15 & $1.22(0.62-2.40)$ & 0.12 \\
\hline $210-260$ & 47 & 17 & $0.94(0.49-1.78)$ & 0.13 \\
\hline$>260$ & 43 & 16 & $0.96(0.49-1.87)$ & 0.13 \\
\hline
\end{tabular}

CI, 95\% confidence interval
WHR (Model 3) or BMI (Model 4) was significantly associated with the investigated genotypes.

\section{Discussion}

The dietary habits can either induce or retard the development of obesity. The selection of macronutrients in the diet appears to be, at least in part, heritable. Excessive food intake is strongly tied to obesity, whereas several studies suggest that a preference for fat and the resulting obesity are also partially genetically determined [5, 6, 23]. In the presented population-based study, we investigated the effects of ID ACE polymorphism on food intake of morbidly obese, obese or non-obese subjects originating from the Czech Caucasian population. In the morbidly obese cohort, we observed that the odds ratio of carrying the DD genotype was approximately fourfold higher in those individuals consuming more than $260 \mathrm{~g}$ of carbohydrates/day, while in the same cohort the odds ratio of carrying the DD genotype in individuals consuming between 210 and $260 \mathrm{~g}$ of carbohydrates/day was 0.27 . It has been reported previously that DD genotype is associated with higher serum ACE level and, therefore, with higher angiotensin II levels, which might be connected to increased susceptibility to high blood pressure and cardiovascular co-morbidities including obesity [4, 7]. In this study, we observed that the morbidly obese individuals with the DD genotype expressed an approximately fourfold risk for their consumption of carbohydrates to be in the highest tertile of the total carbohydrate intake, whereas based on the previously published findings, these morbidly obese DD carriers would be more likely to suffer from high blood pressure and other cardiovascular comorbidities. Thus it could be speculated that at least a proportion of the negative association of the $\mathrm{D}$ allele with cardiovascular health could be due to different native dietary composition or general eating behavior of the individuals. However, it has to be mentioned that the morbidly obese cohort was underpowered for such conclusions and investigation of these associations definitely require further studies on larger population samples.

In the study by Kourlaba et al. [14] carried out on a large population of Greek toddlers and preschoolers, the authors conclude that the total energy intake, as well intake of proteins, was positively associated with obesity-related phenotypes, with these associations being modified by the ID ACE polymorphism. In particular, the authors suggest that the protein intake was associated with being overweight, however, only among the carriers of the D allele (i.e. ID or DD genotypes), which the authors explain on the grounds of higher circulating ACE levels in the DD carriers [25], thus hypothesizing that increased levels of angiotensin II observed in carriers of the D allele may be associated with 
alterations in macronutrient oxidation, thus resulting in fat storage and weight gain. This is partially in accordance with our findings as we observed significantly higher thickness of the supraspinal skin fold in DD carriers compared to carriers of I allele (II + ID). However, in this study, we observed significantly higher prevalence of obesity in II carriers when compared to the D allele carriers (DD + DI), thus suggesting that even though the DD carriers might present with specific anthropometric features (such as distribution of subcutaneous fat), these findings cannot be easily generalized and applied on the prevalence of the complex phenotype of obesity, being in the DD carriers less frequent in our cohort.

Based on our findings, the ID ACE polymorphism could represent a gene modulator of native carbohydrate food intake in both non-obese and obese Czech population, whereas a strong significant effect of DD genotype was observed only in the phenotypes of extreme obesity with the highest tertile of carbohydrate intake.

However, some limitations of our study should be noted. First, the number of morbidly obese subjects (BMI $\geq 40)$ was relatively small ( $n=74)$, thus reducing the statistical power for conclusions about this group. Still, this study is the first study to associate the food intake in the non-obese, obese and morbidly obese adult populations with the ID ACE polymorphism and thus we consider it a pilot one, requiring the subsequent population-based research on a larger population sample. Moreover, the subjects in the study originated from relatively homogeneous Caucasian population, which allowed the described approach. Anyhow, the distributions of SNPs at the whole-genome level should be investigated in various geographic regions before excluding a bias due to population stratification, which might have influenced the results. A family-based association study, such as the transmission disequilibrium test, should also be performed.

Acknowledgments Study was supported by grant of Ministry of Education of the Czech Republic No. 881/2006 and by project of DANONE/2007 by Danone Institute focused on genetic variability of adipokines in obese individuals.

Conflict of interest statement None of the authors had any conflict of interest.

\section{References}

1. Baura GD, Foster DM, Porte D Jr et al (1993) Saturable transport of insulin from plasma into the central nervous system of dogs in vivo: a mechanism for regulated insulin delivery to the brain. J Clin Invest 92(4):1824-1830

2. Bienertova-Vasku J, Bienert P, Tomandl J et al (2008) No association of defined variability in leptin, leptin receptor, adiponectin, proopiomelanocortin and ghrelin gene with food preferences in the Czech population. Nutr Neurosci 11:2-8
3. Bouchard L, Tremblay A, Bouchard C, Pérusse L et al (2007) Contribution of several candidate gene polymorphisms in the determination of adiposity changes: results from the Québec Family Study. Int J Obes (Lond) 31:891-899

4. Cambien F, Poirier O, Lecerf L et al (1992) Deletion polymorphism in the gene for angiotensin-converting enzyme is a potent risk factor for myocardial infarction. Nature 359:641-644

5. Charalambous M, da Rocha ST, Ferguson-Smith AC (2007) Genomic imprinting, growth control and the allocation of nutritional resources: consequences for postnatal life. Curr Opin Endocrinol Diabetes Obes 14:3-12

6. Chung WK, Patki A, Matsuoka N et al (2009) Analysis of 30 genes (355 SNPS) related to energy homeostasis for association with adiposity in European-American and Yup'ik Eskimo populations. Hum Hered 67:193-205

7. Danser AH, Schalekamp MA, Bax WA et al (1995) Angiotensinconverting enzyme in the human heart. Circulation 92:1387-1388

8. de Castro JM (1993) Genetic influences on daily intake and meal patterns of humans. Physiol Behav 53:777-782

9. de Castro JM (2002) Independence of heritable influences on the food intake of free-living humans. Nutrition 18:11-16

10. de Castro JM, Plunkett S (2002) A general model of intake regulation. Neurosci Biobehav Rev 26:581-595

11. Han JC, Rutledge MS, Kozlosky M, Salaita CG et al (2008) Insulin resistance, hyperinsulinemia, and energy intake in overweight children. J Pediatr 152(5):612-617

12. Iimura O, Shimamoto K, Matsuda K et al (1995) Effects of angiotensin receptor antagonist and angiotensin converting enzyme inhibitor on insulin sensitivity in fructose-fed hypertensive rats and essential hypertensives. Am J Hypertens 8:353-357

13. Kinoshita M, Nakaya Y, Harada N et al (2002) Combination therapy of exercise and angiotensin-converting enzyme inhibitor markedly improves insulin sensitivities in hypertensive patients with insulin resistance. Circ J 7:655-658

14. Kourlaba G, Pitsiladis YP, Lagou V et al (2008) Interaction effects between total energy and macronutrient intakes and angiotensin-converting enzyme 1 (ACE) I/D polymorphism on adiposity-related phenotypes in toddlers and preschoolers: the Growth, Exercise and Nutrition Epidemiological Study in preSchoolers (GENESIS). Br J Nutr 100:1333-1340

15. Lagou V, Manios Y, Moran CN et al (2007) Developmental changes in adiposity in toddlers and preschoolers in the GENESIS study and associations with the ACE I/D polymorphism. Int J Obes (Lond) 31:1052-1060

16. Larsson H, Elmståhl S, Berglund $\mathrm{G}$ et al (1998) Evidence for leptin regulation of food intake in humans. J Clin Endocrinol Metab 83:4382-4385

17. Llewellyn $\mathrm{CH}$, van Jaarsveld CH, Boniface D et al (2008) Eating rate is a heritable phenotype related to weight in children. Am J Clin Nutr 88:1560-1566

18. Ma Y, Bertone ER, Stanek EJ 3rd et al (2003) Association between eating patterns and obesity in a free-living US adult population. Am J Epidemiol 158:85-92

19. Moran CN, Vassilopoulos C, Tsiokanos A et al (2005) Effects of interaction between angiotensin I-converting enzyme polymorphisms and lifestyle on adiposity in adolescent Greeks. Obes Res 13:1499-1504

20. Nickenig G, Roling J, Strehlow K et al (1998) Insulin induces upregulation of vascular AT1 receptor gene expression by posttranscriptional mechanisms. Circulation 22:2453-2460

21. Niswender KD, Baskin DG, Schwartz MW (2004) Insulin and its evolving partnership with leptin in the hypothalamic control of energy homeostasis. Trends Endocrinol Metab. 15(8):362-369

22. Ogihara T, Asano T, Ando K et al (2002) Angiotensin II-induced insulin resistance is associated with enhanced insulin signaling. Hypertension 40:872-879 
23. Perrot-Sinal TS (2009) Do these genes make me look fat? Endocrinology 150:1075-1077

24. Rao RH (1996) Pressor doses of angiotensin II increase hepatic glucose output and decrease insulin sensitivity in rats. J Endocrinol 148:311-318

25. Rigat B, Hubert C, Alhenc-Gelas F et al (1990) An insertion/ deletion polymorphism in the angiotensin I-converting enzyme gene accounting for half the variance of serum enzyme levels. J Clin Invest 86:1343-1346

26. Rigat B, Hubert C, Corvol P et al (1992) PCR detection of the insertion/deletion polymorphism in the angiotensin I-converting enzyme gene (DCP1) (dipeptidyl carboxypeptidase. Nucl Acids Res 20:1433

27. Santos JL, Boutin P, Verdich C et al (2006) Genotype-by-nutrient interactions assessed in European obese women: a case-only study: NUGENOB* consortium. Eur J Nutr 45:454-462
28. Shanmugam V, Sell KW, Saha BK (1993) Mistyping ACE heterozygotes. PCR Methods Appl 3:120-121

29. Steinle NI, Hsueh WC, Snitker S et al (2002) Eating behavior in the Old Order Amish: heritability analysis and a genome-wide linkage analysis. Am J Clin Nutr 75:1098-1106

30. Strazzullo P, Iacone R, Iacoviello L et al (2003) Genetic variation in the renin-angiotensin system and abdominal adiposity in men: the Olivetti Prospective Heart Study. Ann Intern Med 138:17-23

31. van den Bree MB, Eaves LJ, Dwyer JT (1999) Genetic and environmental influences on eating patterns of twins aged $>50 \mathrm{y}$. Am J Clin Nutr 70:456-465

32. Wallum BJ, Taborsky GJ Jr, Porte DJ et al (1987) Cerebrospinal fluid insulin levels increase during intravenous insulin infusions in man. J Clin Endocrinol Metab 64(1):190-194 